\title{
Molecular and phylogenetic study of Staphylococcus aureus isolated from human and cattle of Al-Qadisiyah Governorate, Iraq
}

\author{
Ahmed Jasim Neamah ${ }^{1}$, Hayder Naji Ayyez ${ }^{1}$, Saba Falah Klaif ${ }^{1}$, Yahia Ismail Khudhair ${ }^{2}$ and Muthanna Hadi Hussain ${ }^{2}$
}

1. Unit of Zoonotic Diseases, College of Veterinary Medicine, University of Al-Qadisiyah, Al-Qadisiyah, Iraq;

2. Department of Internal and Preventive Medicine, College of Veterinary Medicine, University of Al-Qadisiyah, Al-Qadisiyah, Iraq.

Corresponding author: Muthanna Hadi Hussain, e-mail: muthanna.hussain@qu.edu.iq

Co-authors: AJN: ahmed.neamah@qu.edu.iq, HNA: hayder.ayiz@qu.edu.iq, SFK: Saba.falah@qu.edu.iq,

YIK: yahia.khudiar@qu.edu.iq

Received: 23-04-2019, Accepted: 29-07-2019, Published online: 12-09-2019

doi: 10.14202/vetworld.2019.1378-1382 How to cite this article: Neamah AJ, Ayyez HN, Klaif SF, Khudhair YI, Hussain MH (2019) Molecular and phylogenetic study of Staphylococcus aureus isolated from human and cattle of Al-Qadisiyah Governorate, Iraq, Veterinary World, 12(9): 1378-1382.

\begin{abstract}
Aim: This study was designed to detect the prevalence of Staphylococcus aureus, to estimate the frequency of methicillin resistance gene (mecA), femA (specific gene for $S$. aureus), and lukS gene, and the prevalence of urinary tract infection (UTI) in human and bovine mastitis caused by $S$. aureus.

Materials and Methods: A total of 102 cases of $S$. aureus were included in this study; 72 specimens were isolated from human with UTIs and 30 specimens were isolated from milk of cattle with acute mastitis. Diagnosis was done by VITEK 2 Compact after subculture and purification. All isolates were examined for the presence of mecA, femA, and lukS (PantonValentine leukocidin) using multiplex polymerase chain reaction.

Results: Culture and biochemical evaluation of the samples revealed the presence of $S$. aureus, among which the genes $m e c A$, femA, and lukS were positively detected in $68(94.4 \%), 36(50 \%)$, and $20(27.7 \%)$ of $S$. aureus isolates from methicillin-resistant humans, respectively. In the same manner, the genes $m e c A$, fem $A$, and $\operatorname{lukS}$ were positively detected in $27(90 \%), 14(46.7 \%)$, and $11(36.7 \%)$ of $S$. aureus isolates from methicillin-resistant cattle. Sequencing of partial order of $f e m A$ gene isolated from human isolate and from cattle with $m e c A$ isolated from human revealed high sequence identity with the National Center for Biotechnology Information (NCBI)-Basic Local Alignment Search Tool. S. aureus isolates and the phylogenetic analysis showed that there was a significant genetic similarity ( 0.5 genetic change) between human and animals isolates, and then, the gene sequences were deposited into NCBI-Genbank accession numbers MG696860.1 for $m e c A$ and femA from human, MG696861.1 for $m e c A$ and femA from cattle, MK474469.1 for $m e c A$ and femA gene from human, and MG696862.1 for $m e c A$ and $f e m A$ gene from cattle.

Conclusion: The study represents the first report of genetic relationship between $S$. aureus from humans and cattle of Iraq. Therefore, it is essential to define the role of animals as an important source of the distribution of pathogen related to public health. The continuous monitoring of methicillin susceptibility pattern of $S$. aureus isolates that have high standards of infections might prevent methicillin-resistant $S$. aureus transmission in either direction between human and cattle, the risk of dairy milk on humans, or self-direction between the same species.
\end{abstract}

Keywords: antibiotic, leukotoxin, methicillin, Panton-Valentine, resistance, Staphylococcus aureus.

\section{Introduction}

Since the discovery and extensive utilization of antibiotics, multidrug-resistant strain of Staphylococcus aureus has emerged. Originally, methicillin-resistant S. aureus (MRSA) was discovered in hospitals in the 1960s, which then spread worldwide in community and hospitals in the 1990s, creating reservoirs in both settings [1]. Various infections, ranging from minor skin infections to severe diseases, such as urinary tract infections (UTIs), toxic shock, septicemia, and endocarditis, could be caused

Copyright: Neamah, et al. Open Access. This article is distributed under the terms of the Creative Commons Attribution 4.0 International License (http://creativecommons.org/licenses/ by/4.0/), which permits unrestricted use, distribution, and reproduction in any medium, provided you give appropriate credit to the original author(s) and the source, provide a link to the Creative Commons license, and indicate if changes were made. The Creative Commons Public Domain Dedication waiver (http:// creativecommons.org/publicdomain/zero/1.0/) applies to the data made available in this article, unless otherwise stated. by $S$. aureus. The adaptive power of $S$. aureus to antibiotics produced to the emergence of MRSA. MRSA strain is characterized by its potential to express many virulence factors such as Panton-Valentine leukocidin (PVL). Thus, MRSA is one of the important pathogens implicated in hospital-acquired infection. $S$. aureus is an important pathogen responsible for both nosocomial and community-acquired infections in people and also causes common diseases in animals, especially mastitis. This ubiquitous organism is carried asymptomatically in $20-30 \%$ of the human population [2].

It can lead to a wide range of maladies such as skin and soft tissue infections, mastitis, bone, joint and implant infections, pneumonia, and septicemia. It might be the cause of similar diseases in animals; however, is most economically significant as a cause of bovine mastitis (BM) [3]. The extensive use of antibiotics that accounted for the hasty dissemination of 
antibiotic resistance in $S$. aureus has become a global concern; the most notable example of this phenomenon is MRSA. The first clinical isolate of MRSA was confirmed in 1961, just 1 year after the launch of methicillin, and in the $1980 \mathrm{~s}$, it became a widespread health-care concern $[3,4]$. Methicillin resistance may be attributed to a penicillin-binding protein (PBP) $2 \mathrm{a}$ with an altered $\beta$-lactam affinity $[5,6]$. The mecA gene encodes for PBP 2 a protein, which is carried by a mobile element "Staphylococcal chromosomal cassette (SCC)" and used as a molecular marker for MRSA [7]. Simultaneously, certain factors essential for methicillin resistance $(\mathrm{fem})$ [8] are found in both methicillin-susceptible and MRSA. The part of fem 's factor that combines physically distant from SCC affects the level of the resistance [9]. Integration of the mec complex and neighboring regions into an SCC element was one of the factors contributing to the evolution of mecA-mediated $\beta$-lactam resistance in Staphylococci [10]. MRSA was phenotypically detected in $68.7 \%$ of samples obtained from cattle with mastitis while the methicillin-susceptible S. aureus was detected in $31.3 \%$ of these samples. Genotypic analysis revealed that $54.5 \%$ phenotypically methicillin resistance isolates were mecA gene positive, while $45.5 \%$ of the isolates were $m e c A$ gene negative [11]. This organism is one of the important pathogens in hospital-acquired infection. It has developed resistance to a wide range of antimicrobial drugs, which complicates the treatment of infections. In particular, MRSA has become a disreputable etiological agent for several infections and among the foremost necessary health facility pathogens worldwide [12]. S. aureus is characterized by its several specific virulence factors. The PVL is a major virulence factor related to death lesions of the skin and body covering tissues (e.g., furuncles) and also with community-acquired severe necrotic pneumonia [13]. In humans, $S$. aureus is found in the anterior nares. Nasal carriage of these organisms in hospital staff is a potential source for infection in hospitalized patients, and elimination of nasal carriage has been reported to reduce the incidence of $S$. aureus infections. The antimicrobial resistance profile of human S. aureus is of great public health concern, especially in developing countries where health facilities are inadequate [14].

This study aimed to detect the prevalence of $S$. aureus and the frequency of methicillin-resistant genes and the prevalence of UTI in human and BM caused by $S$. aureus.

\section{Materials and Methods}

\section{Ethical approval}

The Animal Ethical Committee of the College of Veterinary Medicine, University of Al-Qadisiyah, Iraq, has approved this study.

\section{Samples}

Samples were obtained from 72 individuals suffering from UTI at the hospital in Al-Qadisiyah Governorate during January 2016-March 2016, and at the same time, samples were also obtained from 30 cows having acute mastitis.

\section{Bacterial diagnosis}

Bacterial isolates were identified based on cultural and biochemical criteria. The method followed was adapted from a previous study that reported that VITEK2 cards inoculated with fluids sampled directly from culture bottles are suitable for speedy identification and susceptibility testing [15]. A sterile swab or applicator stick was used to transfer a sufficient number of colonies of a pure culture in $3.0 \mathrm{~mL}$ of sterile saline $(0.45 \% \mathrm{NaCl}, \mathrm{pH} 4.5-7.0)$ in a $12 \times 75 \mathrm{~mm}$ clear plastic (polystyrene) test tube. The turbidity was adjusted to $0.50-0.63 \mathrm{Mf}$ and measured using a turbidity meter known as DensiChek [15]. Results were read overnight.

\section{Molecular detection of virulence genes}

Multiplex polymerase chain reaction (PCR) was performed to amplify target genes using primers for the following genes: femA (specific for $S$. aureus), $m e c A$ gene (encoding PBP 2a), and lukS (responsible for the production of PVL). The sequences of these primers are listed in Table-1 $[16,17]$.

\section{DNA extraction of bacterial genome}

Fresh bacterial genomic DNA of $S$. aureus was extracted from nutrient broth samples using Presto ${ }^{\mathrm{TM}}$ Mini Genomic DNA Bacteria Kit (Geneaid, China), according to a previously described method [17]. The extracted DNA was validated by Nanodrop spectrophotometer and stored at $-20^{\circ} \mathrm{C}$ in the refrigerator until further usage.

\section{Polymerase chain reaction (PCR)}

The femA gene (specific to $S$. aureus species), mecA gene (encoding PBP 2a), and $l u k S$ gene (responsible for the production of PVL) were detected using multiplex PCR. The primers were prepared by Bioneer, Korea.

Table-1: Sequences of primers used for multiplex PCR.

\begin{tabular}{|c|c|c|c|c|}
\hline Product genes & Primer name & $5^{\prime}-3^{\prime}$ & Size (bp) & Reference \\
\hline femA & $\begin{array}{l}\text { femA-F } \\
\text { femA-R }\end{array}$ & $\begin{array}{l}\text { CGATCCATATTTACCATATCA } \\
\text { ATCACGCTCTTCGTTTAGTT }\end{array}$ & 450 & {$[17]$} \\
\hline mecA & $\begin{array}{l}\operatorname{mec} A-F \\
\operatorname{mec} A-R\end{array}$ & $\begin{array}{l}\text { ACGAGTAGATGCTCAATATAA } \\
\text { CTTAGTTCTITAGCGATTGC }\end{array}$ & 293 & \\
\hline lukS & $\begin{array}{l}\text { lukS-F } \\
\text { lukS-R }\end{array}$ & $\begin{array}{l}\text { CAGGAGGTAATGGTTCATTT } \\
\text { ATGTCCAGACATTITACCTAA }\end{array}$ & 151 & \\
\hline
\end{tabular}

$\mathrm{PCR}=$ Polymerase chain reaction 


\section{DNA sequencing}

DNA sequencing was performed for confirmative detection of $S$. aureus strain; based on positive detection of $f e m A$ and $m e c A$, two isolates were obtained from humans and one from cattle, and the respective PCR products were purified from agarose gel. The purified fem $A$ and mec $A$ fractions were sent to Bioneer, Korea, for DNA sequencing using Applied Biosystems DNA sequencing system. The genomic sequences were assembled and submitted in GenBank-National Center for Biotechnology Information followed by multiple sequence alignment using Basic Local Alignment Search Tool for phylogenetic tree construction and phylogenetic analysis by MEGA10 program (https://www.megasoftware.net/).

\section{Results}

Culture and biochemical evaluation of the samples revealed the presence of $S$. aureus with positive detection of mecA, femA, and $l u k S$, respectively, in $68(94.4 \%), 36(50 \%)$, and $20(27.7 \%)$ of samples isolated from methicillin-resistant human samples. Similarly, mecA, fem $A$, and $l u k S$ were positively detected, respectively, in 27 (90\%), 14 (46.7\%), and $11(36.7 \%)$ of samples isolated from methicillin-resistant cattle. The MRSA was confirmed by the detection of mec $A$ gene, considered as a genotyping marker, in 68 of $72(94.4 \%)$ samples, while the remaining four isolates were methicillin-sensitive $S$. aureus, although these were methicillin-resistant phenotypically. The multiplex PCR revealed significant differences in the band sizes of mecA, fem A, and $\operatorname{lukS}$ (450, 293, and $151 \mathrm{bp}$, respectively) as illustrated in Figure-1.

The results of nucleotide sequencing of human and cattle MRSA isolates revealed the sequences of targeted genes (mec $A$ and fem $A$ partial gene) that were recorded in Genbank with accession numbers MG696860.1 and MK474469.1 for human isolates and with accession numbers MG696861.1 and MG696862.1 for bovine milk isolates, respectively. Comparative analysis of nucleotide sequences from Iraq samples with the number of global strains present in the Genbank database is shown in Figure-2.

\section{Discussion}

$S$. aureus has been recognized as an important pathogen that causes serious problems in community and hospitalized patients [18]. UTI is the most consequence of common community and hospital bacterial infection and characterized by high rate of treatment failure and high prevalence of $S$. aureus [19].

About $36.3 \%$ of clinical samples isolated from patients with respiratory tract infections in Shiraz province were positive for MRSA, among which the most commonly detected antibiotic resistance genes were tetK $(89.18 \%)$ and mecA $(71.62 \%)$ [20], which suggested that MRSA has more affinity to the UIT than the respiratory infection with the similarity in the presence of the résistance genes, especially mecA. In a total of 152 studies on the community-associated MRSA; the highest prevalence was found in India (16.5-23.5\%) followed by Vietnam $(7.9 \%)$ and Taiwan (3.5-3.8\%) [21]; the difference of these countries from Iraq may be attributed to the cruel circumstances of people in Iraq through the past few decades. A previous study showed that 81 MRSA strains (71.05\%) were mecA gene positive [22], which was slightly less than the results of our study (94.4\%); it may be because, for the previous study, samples were obtained from multilocal lesions; nasal swab, burns, and urine, while we obtained the samples from only UTI in our study.

On the other side, MRSA is highly prevalent in cattle causing mastitis, the most common cattle infection, which leads to a huge economic loses. Hence, the use of right antibiotic in the treatment and control of BM caused by $S$. aureus is crucial [11]. This corroborated with the results found in our study, in which $S$. aureus was detected. This study focused on mastitis associated with $S$. aureus as an important cattle disease with a high incidence in buffaloes, as reported previously $[23,24]$

This study was designed to provide information about the distribution of MRSA based on genotypic marker, mecA, among UTI patients in Al-Qadisiyah Governorate and phylogenic relationship with MRSA isolated from milk obtained from cattle. The gene mecA was detected in $94.4 \%$ of oxacillin-resistant

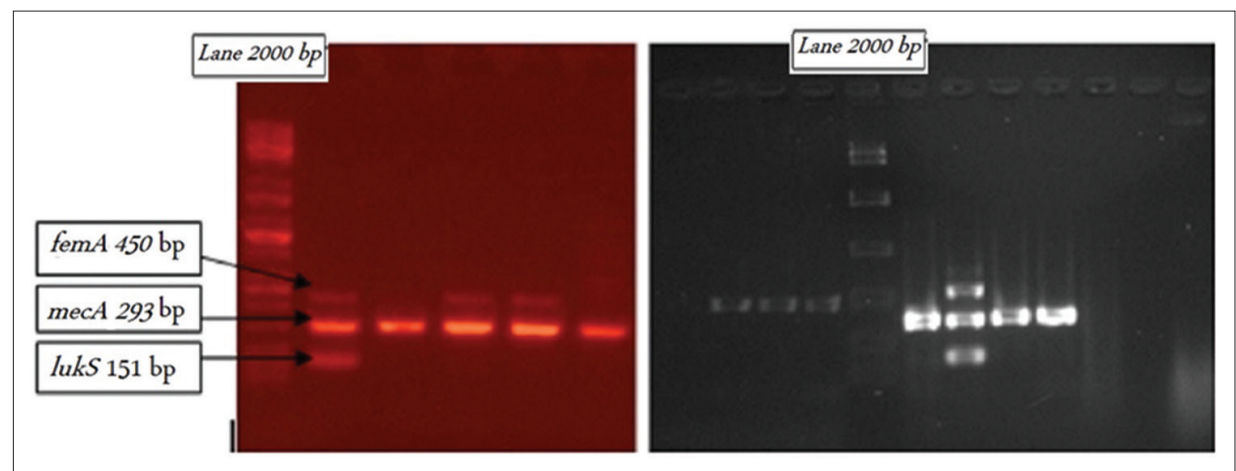

Figure-1: Multiplex polymerase chain reaction for the detection of three genes: femA (450 bp), mecA (293 bp), and lukS (151 bp). 


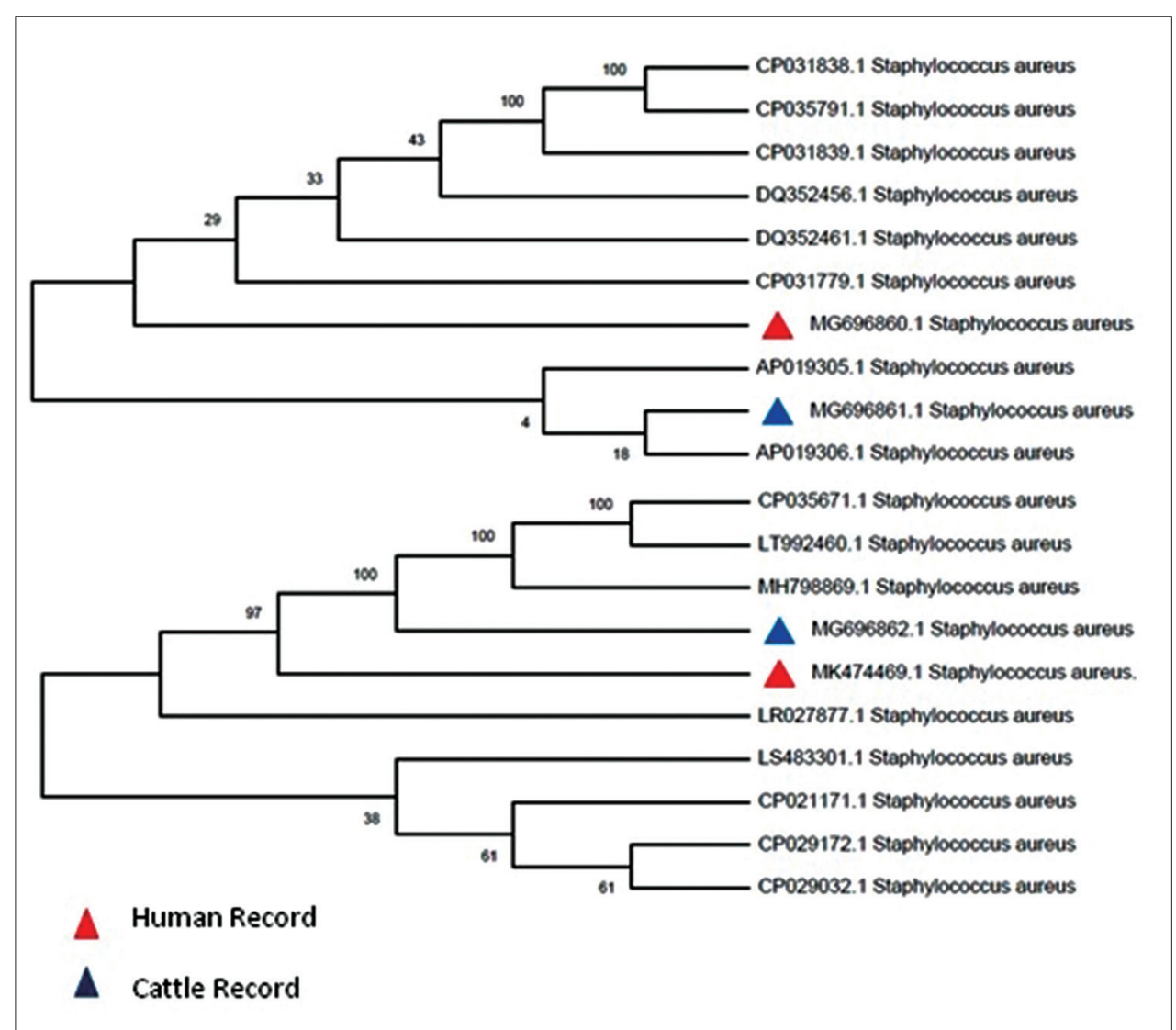

Figure-2: Genetic tree interpretation based on mecA and femA gene partial sequences used for methicillin-resistant Staphylococcus aureus genetic changes according to host and regions.

$S$. aureus [18] which reveals it to be a potential genotypic marker for this criterion.

Among 72 isolates used in this study, $50 \%$ of the isolates did not harbor the gene $f e m A$ and these isolates might possess $f e m B$ marker. The results revealed that $29.4 \%$ of $m e c A$-positive samples expressed $l u k S$ gene and all were positive for $f e m A$, indicating the correlation between the presence of lukS and the presence of fem $A$ because all the isolates that did not possess fem $A$ also lacked $l u k S$ gene. There was high variation for this gene among MRSA isolates. In India, a previous study reported that $85.1 \%$ of MRSA expressed PVL gene [25], while another study reported a prevalence of $64 \%$ for lukS gene among MRSA samples [26]. Such differences in the results were attributed to the geographical variation.

To determine the degree of relatedness between the two clinically isolated local strains of $S$. aureus from human and cattle, phylogenetic trees for fem $A$ and $m e c A$ were constructed based on each gene separately (Figure-2). The sequences of fem $A$ from the two clinically isolated animal and human strains belonged to Clade I and Clade II, respectively. On the contrary, mecA sequences from the two clinically isolated animal and human strains belonged to the same clade. The present data suggested the novelty of the two clinically isolated animal and human strains. This result indicates the indispensable need to employ an Multilocus sequence type (ST) approach in the future analyses to assign the ST of each strain and the likelihood of raising a novel ST from the strains under study.

\section{Conclusion}

$S$. aureus is recognized as an important pathogen that causes serious problems in community, hospitalized patients, and animals. The present data suggested the novelty of the two clinically isolated local strains from animal and human. Consequently, the continuous monitoring of methicillin susceptibility pattern of $S$. aureus isolates that have high standards of infections might help in the prevention of MRSA transmission in either direction between human and cattle, the risk of dairy milk on humans, or self-direction between the same genus.

\section{Authors' Contributions}

YIK and HNA proposed the hypothesis. AJN designed the study. AJN and HNA collected the samples from human while the samples were collected from cattle by YIK and MHH. AJN and SFK conducted the experiments. All authors collected the references, read and approved the final manuscript after editing by $\mathrm{MHH}$.

\section{Acknowledgments}

The authors would like to thank the staff of Al-Qadisiyah hospital. The owners of the animals were very helpful and facilitated sample collection from animals. This study was self-funded. 


\section{Competing Interests} interests.

The authors declare that they have no competing

\section{Publisher's Note}

Veterinary World remains neutral with regard to jurisdictional claims in published institutional affiliation.

\section{References}

1. Deleo, F.R. and Chambers, H.F. (2009) Re-emergence of antibiotic-resistant Staphylococcus aureus in the genomics era. J. Clin. Invest., 119(9): 2464.

2. Van Belkum, A. (2000) Molecular Epidemiology of Methicillin-Resistant Staphylococcus aureus Strains: State of Affairs and Tomorrow's Possibilities. Microbial Drug Resistance. 6(3):173-88.

3. Holmes, M.A. and Zadoks, R.N. (2011) Methicillin-resistant S. aureus in human and bovine mastitis. J. Mammary Gland Biol. Neoplasia, 16(4): 373-382.

4. Livermore, D.M. (2000) Antibiotic resistance in staphylococci. Int. J. Antimicrob. Agents, 16(1): 3-10.

5. Georgopapadakou, N.H. and Liu, F.Y. (1980) Binding of $\beta$ - lactam antibiotics to penicillin-binding proteins in a Staphylococcus aureus and Staphylococcus faecalis: Relation to antibacterial activity. J. Antimicrob. Chemother., 18(5): 834-836.

6. Hartman, B.J. and Tomasz A. (1986) Expression of methicillin resistance in heterogeneous strains of Staphylococcus aureus. J. Antimicrob. Chemother., 29(1): 85-92.

7. Hiramatu, K., Cui, L., Kurida, M. and Ito, T. (2001) The emergence and evolution of methicillin-resistant Staphylococcus aureus. Trends Microbiol., 9(10): 486-493.

8. Hussain, S.F., Khatoon, A., Shahid, S.M., Ismail, M. and Azhar, A. (2013) Discriminative multiplex (hexaplex) PCR strategy for the detection of methicillin resistance and virulence factors in Staphylococcus aureus. Afr. J. Microbiol. Res., 7(3): 196-201.

9. Berger, B.B., Barberis, M.L., Striissle, A. and Kayser, F.H. (1989) Fem a: A host-mediated factor essential for methicillin resistance in Staphylococcus aureus: Molecular cloning and characterization. Mol. Gen. Genet., 219(1-2): 263-269.

10. Maria, M. (2018) Factors contributing to the evolution of mecA-mediated b-lactam resistance in Staphylococci: Update and new insights from whole-genome sequencing (WGS). Front. Microbiol., 9(1): 1-16.

11. Elkenany, R.M. (2018) Genetic characterization of enterotoxigenic strains of methicillin resistance and susceptible $S$. aureus recovered from bovine mastitis. Asian J. Biol. Sci., 11(1): 1-8.

12. Nour, M., Mastouri, M. and Nejme, M.B. (2005). Methicillinresistant Staphylococcus aureus: Emergence and molecular basis of resistance. Pathol. Biol., 53(6): 334-340.

13. Lina, G., Piemont, Y., Godail-Gamot, F., Bes, M., Peter, M.O. Gauduchon, V., Vendenesch, F. and Etienne, J. (1999) Involvement of panton-valentine leukocidin producing Staphylococcus aureus in primary skin infections and pneumonia. Clin. Infect. Dis., 29(5): 1128-1132.

14. Von-Eiff, C., Becker, K., Machka, K., Stammer, H. and Peters G. (2001). Nasal carriage as a source of Staphylococcus aureus bacteremia. N. Engl. J. Med., 344(1): 11-16.

15. Nimer, N.A., Al-Saa'da, R.J. and Abuelaish, O. (2016) Accuracy of the VITEK 2 system for a rapid and direct identification and susceptibility testing of gram-negative rods and gram-positive cocci in blood samples. East. Mediterr. Health J., 22(3): 193-200.

16. Al-Talib, H., Yean, C.Y., Al-Khateeb, A., Hassan, H., Singh, K.K., Al-Jashamy, K. and Ravichandran, M. (2009) A pentaplex PCR assay for the rapid detection of methicillin-resistant Staphylococcus aureus and panton-valentine leucocidin. BMC Microbiol., 9: 113.

17. Psifidi, A., Dovas, C.I., Bramis, G., Lazuo, T., Russel, C.L., Arsenos, G. and Banos, G. (2015) Comparison of eleven methods for genomic DNA extraction suitable for largescale whole-genome genotyping and long-term DNA banking using blood samples. US Natl. Libr. Med. Natl. Inst. Health, 10(1): e0115960.

18. Jan, M.B., Turnidge, J.D. and Sentry, A. (2002) High prevalence of Oxacillin-resistant $S$. aureus isolates for hospitalized patients in Asia-Pacific and South Africa. The sentry antimicrobial surveillance program, 1998-1999. J. Antimicrob Chemother., 46(3): 879-887.

19. Alsamarai, A.M., Shler, A.K. and Hajer, A. (2017) Urinary tract infection in female in Kirkuk city, Iraq: Association between risk factors and bacterial type. Our Dermatol. Online J., 8(3): 242-249.

20. Banafshe, D., Soheila, S., Ebrahim, K. and Ladan A. (2015) Methicillin-resistant Staphylococcus aureus isolated from various types of hospital infections in pediatrics: Pantonvalentine leukocidin, Staphylococcal chromosomal cassette mec SCCmec phenotypes and antibiotic resistance properties. Jundishapur J. Microbiol., 8(11): e11341.

21. Wong, J.W., Ip, M., Tang, A., Wei, V.W., Wong, S.Y., Riley, S., Read, J.M. and Kwok, K.O. (2018) Prevalence and risk factors of community-associated methicillin-resistant $S$. aureus carriage in Asia-Pacific region from 2000 to 2016: A systematic review and meta-analysis. Clin. Epidemiol. J., 10(10): 1489-1501.

22. Al-Marzoqi, A.H., Azize, H., Al-Dulaimi, T. and Nada, A. (2014) Phenotypic detection of resistance in Staphylococcus aureus isolates: Detection of (mec A and fem A) gene in methicillin-resistant Staphylococcus aureus (MRSA) by polymerase chain reaction. J. Nat. Sci. Res., 4(2): 112-118.

23. Hamed, M.I. and Zaitoun, A.M. (2014) Prevalence of S. aureus subclinical mastitis in dairy buffaloes farms. Int. J. Livest. Res., 4(3): 21-28.

24. Prabhu, K.N., Ruban, W.S., Kumar, G.S., Sharada, R. and Padalkar, R.D. (2015) Sub-clinical mastitis in buffaloes: Prevalence, isolation and antimicrobial resistance of S. aureus. Buffalo Bull., 34(2): 215-222.

25. Kaur, H., Purwar, S., Saini, A., Kaur, H., Karadesai, S.G., Kholkute, S.D. and Roy, S. (2012) Status of methicillin-resistant $S$. aureus infection and evaluation of PVL producing strains in Belgaum, South India. J. Krishna Inst. Med. Sci. Univ., 1(2): 43-51.

26. Souza, N.D., Rodrigues, C. and Mehta, A. (2010). Molecular characterization of methicillin-resistant $S$. aureus with emergence of epidemic clones of sequence type (ST) and ST 772 in Mumbai. India J. Clin. Microbial., 48(5): 1806011. 